\title{
Ability of the Short Physical Performance Battery Frailty Index to Predict Mortality and Hospital Readmission in Patients with Liver Cirrhosis
}

\author{
Mervat Essam Behiry (D), Sherif Mogawer, Ahmed Yamany, Maha Rakha, Rana Awad, \\ Nahla Emad, and Yasmine Abdelfatah
}

School of Medicine, Cairo University, Egypt

Correspondence should be addressed to Mervat Essam Behiry; lambaa2020@yahoo.com

Received 18 December 2018; Accepted 27 March 2019; Published 2 May 2019

Academic Editor: Simon Bramhall

Copyright (C) 2019 Mervat Essam Behiry et al. This is an open access article distributed under the Creative Commons Attribution License, which permits unrestricted use, distribution, and reproduction in any medium, provided the original work is properly cited.

\begin{abstract}
Background/Aims. Unplanned hospitalisation is a marker of poor prognosis and a major financial burden in patients with cirrhosis. Frailty-screening tools could determine the risk for unplanned hospital admissions and death. The study aims to evaluate the bedside frailty-screening tool (Short Physical Performance Battery (SPPB)) in prediction of mortality in patients with liver cirrhosis. Methods. One hundred forty-five patients with liver cirrhosis were recruited from Cairo University Hospital. Clinical assessment and routine laboratory tests were performed, and the SPPB frailty index, Child score, and model for end-stage liver disease (MELD) score were calculated on admission. These metrics were compared to assess mortality outcomes over the course of 90 days. Results. The mean age of the patients was $60 \pm 7$ years, and frailty index score (SD) was $6 \pm 3$. The overall 90 -day readmission rate was $43.4 \%$, while the overall 90 -day mortality rate was $18.6 \%$. SPPB scores differed significantly between survivors (4.1 \pm 1.4$)$ and nonsurvivors $(6.47 \pm 2.8)(\mathrm{P}$ value $\leq 0.001)$ as well as between readmitted patients $(7.5 \pm 2.9)$ and patients who were not readmitted $(4.5 \pm 1.9)$ (P value $\leq 0.001$ ), while the Child and MELD scores showed no associations with patient outcomes. SPPB performed better with a specificity of $72.3 \%$ and a sensitivity of $72.2 \%$ for predicting mortality. Conclusions. SPPB could be a screening tool used to detect frailty and excelled over traditional scores as a predictor of death. A low SPPB frailty score among hospitalised patients with cirrhosis is associated with poor outcomes.
\end{abstract}

\section{Introduction}

Frailty is defined by decreased strength, power, and diminished physiological function that in turn leads to increased physical dependency and increased risk of mortality especially in older age and those with debilitating diseases [1].

Posthepatitic liver cirrhosis has a high prevalence in Egypt. A comprehensive assessment of hepatitis $\mathrm{C}$ virus (HCV) epidemiology was conducted in 2018, revealing high incidence and prevalence levels across all populations in Egypt. The pooled mean HCV prevalence was estimated to be $11.9 \%$ in the general population, $55.6 \%$ among populations at high risk, $14.3 \%$ among populations at intermediate risk, and $56.0 \%$ among populations with liver-related conditions including liver cirrhosis [2].

HCV-related cirrhosis is strongly associated with protein energy malnutrition (PEM), sarcopenia, frailty, and physical atrophy. This was found to be caused by the release of musclewasting cytokines, the derangement of muscle proteins, and the increased autophagy of muscles, all of which are mediated by elevated levels of tumour necrosis factor, elevated concentrations of ammonia, and impaired ureagenesis [3].

The assessment of physical frailty in patients who have undergone liver transplantation has been widely discussed. A strong association between frailty and poor outcomes after transplantation has been reported [4]. The six-minute walk test has been indicated to be a surrogate test for the pretransplant evaluation of functional capacity and a significant determinant of posttransplantation survival [5].

Unplanned hospitalisation is a major risk factor for poor prognosis and increased. Frailty is considered an independent predictor of unplanned hospitalisations or death in cirrhotic outpatients [6]. 
Given the importance of the detection of frailty and early intervention in cirrhotic patients, many studies have addressed the potential effects of interventional exercise and nutritional supplement strategies [7-9]. These strategies may improve physical function and quality of life and accordingly the frailty index. This would likely decrease the possibility of cirrhosis-associated morbidities, unplanned hospital readmission, health care-related costs, and death [10].

Multiple clinical models of frailty have been proposed that use combinations of different parameters such as the Clinical Frailty Scale (CFS) [11], the Model for End-Stage Liver Disease-Sodium score [12], the activities of daily living (ADL) score, the Braden Scale [13], and the Fried Frailty Index [14]. The Short Physical Performance Battery (SPPB) has emerged as one of the most promising tools for evaluating functional capability. It has proven to provide standard parameters that can be used uniformly across clinical and research settings with high predictiveness for disability onset and adverse outcomes, especially in older patients [15].

In our study, the main objective was to screen for and evaluate frailty among cirrhotic Egyptian patients using the SPPB and to determine the impact of frailty on hospital readmission and mortality.

\section{Methods}

2.1. Study Design and Sample Population. Our cohort study included 145 Egyptian patients with liver cirrhosis. This study was conducted at Kasr Al-Aini Hospital. Patients with posthepatitic cirrhosis who were $\geq 18$ years old were selected from the internal medicine wards, while patients with current hepatic or extrahepatic malignancies; patients with overt hepatic encephalopathy; patients in comas; patients with any medical, physical, neurological disabilities; and patients who used medications (sedatives and anticonvulsants) that compromised their balance were excluded from the study.

Full history and clinical examination were done for all patients.

2.1.1. Anthropometric Assessments. Weight and body mass index (BMI) were measured for each patient.

2.1.2. Blood Collection and Sample Preparation. Ten millilitres of blood was withdrawn from each subject. The complete blood count was estimated using a cell counter with a Cell Dyn machine. The estimation of the levels of serum creatinine and liver enzymes was performed using a kinetic method via an automated Dimension system. The serum levels of albumin, prothrombin concentration (PC), the international normalized ratio (INR) for prothrombin time, and the thyroid profile were also determined.

2.1.3. Model End-Stage Liver Disease MELD Score Evaluation. The MELD score uses the patient's serum levels of bilirubin and creatinine and their INR to predict survival [16]. It is calculated according to the following formula:

MELD $=3.78 \times \ln [$ serum bilirubin $(\mathrm{mg} / \mathrm{dL})]+11.2$ $\times \ln [\mathrm{INR}]+9.57 \times \ln [$ serum creatinine $(\mathrm{mg} / \mathrm{dL})]+6.43$.
According to their MELD score " 20 " patients were classified into the following two groups:

Group (A): patients with MELD scores $>20$.

Group (B): patients with MELD scores $\leq 20$.

2.1.4. Child-Turcotte-Pugh (CTP) Classification. The CTP score combines five clinical measures of liver disease. Each measure is scored from 1 to 3 , with 3 indicating the most severe level of derangement. Patients with chronic liver disease are classified as Child-Pugh classes A to C [17].

2.1.5. Frailty Assessment according to the Short Physical Performance Battery (SPPB). The SPPB is a functional test that measures gait speed ( 8 -foot walk), standing balance, and lower extremity strength and power (via a task involving rising from a chair). The average of three trials was used. Each test was scored on a scale from 0 to 4 points, with a total score range of 0 to 12 points [18]. The patients were contacted after three months to determine the outcomes of mortality or readmission to the hospital.

2.1.6. Handgrip Assessment. The purpose of this test is to measure the maximum isometric strength of the hand and forearm muscles. Each subject holds a handgrip dynamometer (Lafayette, USA) in his /her hand, with the arm at a right angle to his/her body and the elbow held by their side. The best of three attempts, with 30 seconds of rest between the trials, for each hand was recorded in kilograms to one decimal point [19].

2.2. Data Management and Statistical Analysis. Data were precoded and entered in Microsoft Excel. Quantitative variables are presented as the mean (SD). Qualitative variables are described as numbers and percentages. A chi-square test was used to compare qualitative variables between groups. An unpaired t-test was used to compare quantitative variables in the parametric data $(\mathrm{SD}<50 \%$ mean). A paired t-test was used to compare quantitative variables within the same group. A P value $<0.05$ was considered significant. Pearson's correlation was used to test for statistically significant associations. The data have been tabulated for visualization.

2.3. Ethical Considerations. Written informed consent was obtained from all subjects. The study protocol maintained patient confidentiality and conformed to the standards of the Declaration of Helsinki; the study protocol was revised, accepted, and approved by the internal review board (Ethical Committee of Internal Medicine, Faculty of Medicine, Cairo University, protocol number 16-2017-/2018 on the date of 26/8/2017).

\section{Results}

3.1. Demographic and Laboratory Data. In our study, the mean age of the patients was $60 \pm 7$ years, ranging from 36 to 80 years. Seventy-five out of 145 patients were male. The mean (SD) MELD score was $16 \pm 6$, while the mean CTP score was $10 \pm 2$. The demographics, clinical characteristics, and laboratory data are shown in Table 1. 
TABLE 1: Demographic, clinical, and laboratory data of the studied population.

\begin{tabular}{|c|c|}
\hline Variables & $\mathrm{N}=145$ \\
\hline Age $\left(\right.$ mean $\left.\pm \mathrm{SD}^{*}\right)$ & $60 \pm 7$ \\
\hline \multicolumn{2}{|l|}{ Gender (N, \%) } \\
\hline Male & $75(51.7 \%)$ \\
\hline Female & $70(48.3 \%)$ \\
\hline Body mass index (mean $\pm \mathrm{SD})$ & $24.2 \pm 3.4$ \\
\hline $\mathrm{CPT} \dagger$ score $($ mean $\pm \mathrm{SD})$ & $10 \pm 2$ \\
\hline Class (A) & $12(8.3 \%)$ \\
\hline Class(B) & $50(34.5 \%)$ \\
\hline Class $(\mathrm{C})$ & $83(57.2 \%)$ \\
\hline MELD $\ddagger$ Score $($ mean \pm SD) & $16 \pm 6$ \\
\hline MELD > 20 & $15(10 \%)$ \\
\hline MELD $\leq 20$ & $130(90 \%)$ \\
\hline \multicolumn{2}{|l|}{ Comorbidities $(\mathrm{N}, \%)$} \\
\hline Diabetes Mellitus & $45(31 \%)$ \\
\hline Hypertension & $22(15.2 \%)$ \\
\hline Hematemesis (N, \%) & $28(19.3 \%)$ \\
\hline $\mathrm{Hb}^{\#} \mathrm{gm} / \mathrm{dl}($ mean $\pm \mathrm{SD})$ & $9.11 \pm 1.71$ \\
\hline Albumin gm/dl (mean \pm SD) & $2.4 \pm 0.5$ \\
\hline Creatinine mg/dl $($ mean $\pm \mathrm{SD})$ & $1.4 \pm 0.3$ \\
\hline Total Bilirubin mg/dl $($ mean \pm SD) & $1.7 \pm 0.9$ \\
\hline $\mathrm{ALT}^{\S} \mathrm{IU} / \mathrm{L}($ mean $\pm \mathrm{SD})$ & $51 \pm 97$ \\
\hline AST $^{g}$ IU/L (mean \pm SD) & $92 \pm 198$ \\
\hline $\mathrm{INR}^{\dagger \dagger}($ mean $\pm \mathrm{SD})$ & $1.61 \pm 0.91$ \\
\hline $\mathrm{TSH}^{* *} \mathrm{IU} / \mathrm{L}($ mean $\pm \mathrm{SD})$ & $1.99 \pm 1.3$ \\
\hline $\mathrm{CrP}^{\ddagger \ddagger}(\mathrm{mg} / \mathrm{dl})$ & 22.6 \\
\hline Hand grip score & $14.9 \pm 5.6$ \\
\hline Short Physical Performance Battery & $6 \pm 3$ \\
\hline Hospital readmission (3 months) & $63(43.4 \%)$ \\
\hline \multicolumn{2}{|l|}{ Patient survival (3 months) } \\
\hline Survivors (N, \%) & $118(81.4 \%)$ \\
\hline Non-survivors (N, \%) & $27(18.6 \%)$ \\
\hline \multicolumn{2}{|c|}{$\begin{array}{l}{ }^{*} \text { SD standard deviation: } *, \dagger, \ddagger, \S, I I, 9 \text {, and } \# \text {. } \\
{ }^{\dagger} \mathrm{CPT} \text { : Child-Turcotte-Pugh; }{ }^{\ddagger} \text { MELD: model for end stage liver dis- } \\
\text { ease; }{ }^{\S} \text { ALT: alanine transaminase; } 9 \text { AST: aspartate transaminase; }{ }^{\#} \mathrm{Hb} \\
\text { hemoglobin; }{ }^{* *} \text { TSH: thyrotropin stimulating hormone; }{ }^{\dagger \dagger} \text { INR: international } \\
\text { normalized ratio; }{ }^{*} \text { CrP: C reactive protein. }\end{array}$} \\
\hline
\end{tabular}

3.2. Readmission and 90-Day Mortality. The overall 90-day readmission rate was $43.4 \%$ and it mainly occurred due to cirrhosis-related complications, including hematemesis (28 patients), in addition to hepatic coma (19 patients), ascites necessitating tapping (10 patients), and comorbidities-related (8 patients). The overall 90 -day mortality reaches $18.6 \%$.

3.3. Correlations with the SPPB Frailty Score. The data revealed that there was a significant negative correlation between the frailty score assessed by the SPPB and age, CTP score, and MELD score $(r=-0.428,-0.509$, and -0.262 and $\mathrm{p}=0.001,0.001$, and 0.047 , respectively). Frailty had a positive correlation with the handgrip test score $(r=0.568$ and $\mathrm{p}=0.001$ ), as shown in Table 2 .
TABLE 2: Correlation between Frailty score and other parameters.

\begin{tabular}{|c|c|c|}
\hline \multirow{2}{*}{ Variable } & \multicolumn{2}{|c|}{ Frailty score } \\
\hline & R-value & p-value \\
\hline Age & -0.428 & 0.001 \\
\hline BMI $^{*}$ & 0.134 & 0.315 \\
\hline Hemoglobin $(\mathrm{Hb} \%)$ & 0.065 & 0.630 \\
\hline Albumin & 0.333 & 0.011 \\
\hline $\mathrm{ALT}^{\dagger}$ & 0.058 & 0.664 \\
\hline $\mathrm{AST}^{\ddagger}$ & 0.088 & 0.512 \\
\hline $\mathrm{CrP}^{\S}$ & -0.182 & 0.173 \\
\hline Hand grip & 0.568 & 0.001 \\
\hline CTP's score & -0.509 & 0.001 \\
\hline MELD $^{\#}$ score & -0.262 & 0.047 \\
\hline
\end{tabular}

${ }^{*} \mathrm{BMI}$ : body mass index; ${ }^{\dagger} \mathrm{ALT}$ : alanine transaminase; ${ }^{\ddagger} \mathrm{AST}$ : aspartate transaminase; ${ }^{\varsigma} \mathrm{CrP}$ : C reactive protein; ${ }^{9} \mathrm{CPT}$ : Child-Turcotte-Pugh; ${ }^{\#}$ MELD: model for end stage liver disease.

The results demonstrated that the overall 90-day readmission occurred in patients with mean frailty score of $4.88 \pm$ 1.96, MELD score of $16.18 \pm 6.72$, CTP score of $9.88 \pm 1.55$, and handgrip score of $14.012 \pm 5.7$, and those with the overall 90-day mortality had mean frailty score of $4.18 \pm 1.47$, MELD score of $17.27 \pm 9.5$, CTP score of $9.64 \pm 1.28$, and handgrip score of $12.909 \pm 5.39$

The frailty score was significantly higher in males $(6.83 \pm$ $2.866)$ than in females $(5.18 \pm 2.420)(\mathrm{p}=0.021)$. The frailty score was $4.88 \pm 1.96$ in patients who were readmitted to the hospital, compared with $7.56 \pm 2.95$ in those who were not readmitted to the hospital ( $\mathrm{p}$ value $<0.001$ ). Survivors had significantly higher frailty scores than nonsurvivors $(6.47$ \pm 2.8 versus $4.18 \pm 1.47$, respectively; $\mathrm{p} \leq 0.001$ ); however frailty scores did not differ between patients with and without comorbidities.

The data showed that patients with lower frailty scores $(4.88 \pm 1.965)$ had a higher risk of hospital readmission than those with higher scores $(7.56 \pm 2.959)(\mathrm{p} \leq 0.001)$.

Ninety-day mortality was associated only with older age; the mean age of survivors was $59.17 \pm 6.907$ years, while the mean age of nonsurvivors was $64.36 \pm 7.474$ years ( $\mathrm{p}$ value $=0.031)$. However, no significant differences in mortality were observed with regard to sex, BMI, the presence of comorbidities, or laboratory profiles.

Hospital readmission was not associated with any demographic, laboratory, or clinical parameters.

There was no significant difference in patient survival and hospital readmission based on MELD, CTP, or handgrip scores.

3.4. The Sensitivity and Specificity of Frailty Score in Prediction of Increased Mortality and 90-Day Readmission. The ROC curve analyses of mortality based on frailty, handgrip, MELD, and CTP scores revealed that only frailty score had a significant area under the curve (AUC) $(0.743$; p value $=0.013$ ). Frailty scores had fair sensitivity and specificity $(72.7 \%$ and $72.3 \%$, respectively) at a criterion of 4.50 with a $95 \%$ CI of 0.603-0.883 as shown in Figure 1. 
TABLE 3: Results of ROC curve analyses of predictors of hospital readmission.

\begin{tabular}{lcccccccr}
\hline Variable(s) & AUC* & Cut-off point & P value & Sensitivity & Specificity & PPV & NPV & $\begin{array}{c}\text { Asymptotic 95\% } \\
\text { Confidence Interval }\end{array}$ \\
\hline frailty index & 0.383 & 5.50 & 0.136 & $45.5 \%$ & $58.3 \%$ & $40.0 \%$ & $63.6 \%$ & 0.236 \\
\hline hand grip & 0.460 & 14.50 & 0.614 & $45.5 \%$ & $47.2 \%$ & $34.5 \%$ & $58.6 \%$ & 0.529 \\
\hline $\mathrm{CTP}^{\dagger}$ score & 0.612 & 9.50 & 0.156 & $40.9 \%$ & $47.2 \%$ & $32.1 \%$ & $56.7 \%$ & 0.466 \\
\hline $\begin{array}{l}\mathrm{MELD}^{\ddagger} \\
\text { score }\end{array}$ & 0.522 & 15.50 & 0.779 & $50.0 \%$ & $50.0 \%$ & $37.9 \%$ & $62.1 \%$ & 0.619 \\
\hline
\end{tabular}

${ }^{\dagger}$ AUC: area under curve; ${ }^{\dagger} \mathrm{CPT}$ : Child-Turcotte-Pugh; ${ }^{\ddagger} \mathrm{MELD}$ : model for end stage liver disease.

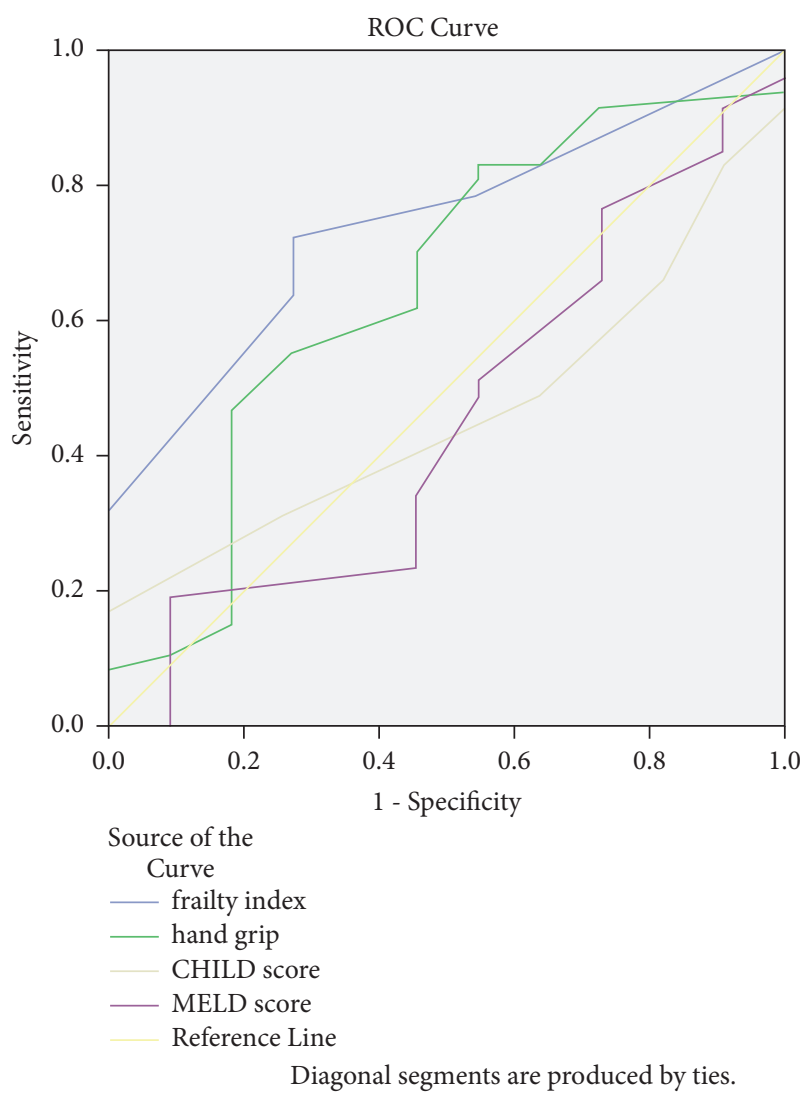

FIGURE 1: ROC curve for mortality predictors among inpatients with liver cirrhosis.

However none of the before-mentioned parameters were potential predictors of hospital readmission (Table 3).

\section{Discussion}

The current results revealed that a lower frailty score was associated with hospital readmission and mortality. The objective of the current study was to screen for frailty among cirrhotic hospitalised patients and evaluate the role of frailty in unplanned hospital readmission and increased mortality.

There are established models for predicting the risk of poor outcomes such as CTP and MELD scores, yet their discriminative abilities are controversial [20]. Moreover, the frailty assessment is not included [21]. Factors associated with increased sarcopenia and cirrhosis include older age, increased severity of the associated liver disease, the presence of other chronic comorbidities, and longer duration of endstage liver disease [22].

Frailty mainly contributes to malnutrition, which is prevalent in $60 \%$ of end-stage liver disease patients. This is due to poor dietary intake, anorexia, fat malabsorptionassociated disorders such as chronic pancreatitis, and disrupted hepatic metabolism [23, 24].

In this study, the SPPB was used to assess frailty. The benefits of this assessment tool include good reliability, validity, and responsiveness as well as simplicity. In addition, the SPPB only requires 5 to 10 minutes to complete, so it can be integrated into patient management without excessive time consumption [25].

In the present study, the mean (SD) score on the SPPB, which combines the results of gait speed, chair standing, and balance tests, was $6 \pm 3$. There was a significant negative correlation between frailty as assessed by the SPPB and age, CTP scores, and MELD scores. Frailty index was positively correlated with the handgrip score. Dunn and colleagues reported that, for each $0.10-\mathrm{m} / \mathrm{sec}$ reduction in walking speed, there is a $22 \%$ increase in the number of hospitalised days in frail patients with cirrhosis [26].

The reported readmission estimates were variable, with heterogeneous findings [27] ranging from $10 \%$ to $71 \%$ [2830]. It has been noted that there is a robust relationship between readmission and subsequent mortality [31-33]. The overall 90 -day readmission rate was $43.4 \%$, while the overall 90 -day mortality rate was $18.6 \%$, and the most common cause of readmission in our population was cirrhosis-related complications. Haematemesis was the major cause of readmission, followed by hepatic encephalopathy [27]. Few studies have noted that increased readmission among cirrhotics is more often seen in those with diabetes. Diabetes is associated with a greater than $70 \%$ readmission rate due to increased incidence of infections and renal impairment [34, 35].

In the current study, the frailty scores were lower in patients with unplanned hospital readmission and in nonsurvivors. Ninety-day mortality was associated only with older age. There were no significant differences in mortality with regard to sex, BMI, the presence of comorbidities, or laboratory profiles. Hospital readmission was not associated with any demographic, laboratory, or clinical parameters.

Although MELD and CTP scores are disease-specific measurements that are commonly used in the care of patients with cirrhosis, neither MELD nor CTP scores differed between survivors and nonsurvivors or between patients 
who were readmitted to hospital and those who were not. This finding might be explained by the fact that the MELD score depends mainly on dynamic parameters that could be affected by laboratory variation. Some variables of the CTP score (hepatic encephalopathy, ascites grade, and nutrition) are subjective [13]. In addition, this study had a relatively small sample size, and most of our study population had MELD scores less than " 20 ", reducing the comparative and discriminative abilities of this study.

Attempting to assess predictors of poor outcome, the current study examined the performance of the SPPB, handgrip test, MELD, and CTP. The results revealed that the frailty score is a potential predictor of mortality in cirrhotic patients, with fair sensitivity and specificity and its performance was superior to that of the handgrip test. The SPPB was found to be better at assessing physical function than the handgrip test possibly because it involves more complex coordination and depends on a larger portion of the total body muscle mass.

Regarding hospital readmission, none of the aforementioned measurements affected the 90-day risk of readmission. This finding was contradictory to those of other studies, which demonstrated an association between the gait speed portion of the frailty test and subsequent hospitalisation in elderly people [36-38]. This difference might be attributed to the variation in the characteristics of the different study populations that may have affected their readmission.

The limitations of this study include the small sample size. Secondly, sarcopenia was not assessed, which may infer a causal relation with frailty. However, this study points to an area of future inquiry in our cirrhotic patients.

We recommend further studies with larger populations and the construction of a combined model that merges the different scores and parameters to increase accuracy and precision.

To conclude, frailty is easily assessed by the SPPB which does not require extensive training for clinicians or nurses. A low SPPB frailty score among hospitalised patients with cirrhosis is associated with poor outcomes. The SPPB could serve as a predictor of hospital readmission and increased mortality among cirrhotic patients.

\section{Data Availability}

The data used to support the findings of this study are available from the corresponding author upon request.

\section{Consent}

Informed consent was obtained from all individual participants included in the study.

\section{Disclosure}

This research did not receive any specific grant from funding agencies in the public, commercial, or not-for-profit sectors.

\section{Conflicts of Interest}

The authors declare that they have no conflicts of interest.

\section{Authors' Contributions}

Mervat Essam Behiry, Sherif Mogawer, and Rana Awad designed the study, interpreted data, and wrote the manuscript. Ahmed Yamany, Maha Rakha, Mervat Essam Behiry, and Yasmine Abdelfatah contributed to data interpretation, article reviews, and editing. Yasmine Abdelfatah and Nahla Emad gathered the data of the patients. Sherif Mogawer, Maha Rakha, and Mervat Essam Behiry contributed to study design, data analysis, and interpretation and reviewed and edited the manuscript. Rana Awad, Nahla Emad, and Ahmed Yamany contributed to critical revision of the manuscript for important intellectual content. All authors contributed to study supervision and revised the final form of the manuscript.

\section{Acknowledgments}

We thank all the participants in this work.

\section{References}

[1] J. E. Morley, B. Vellas, G. Abellan van Kan et al., "Frailty consensus: a call to action," Journal of the American Medical Directors Association, vol. 14, no. 6, pp. 392-397, 2013.

[2] S. P. Kouyoumjian, H. Chemaitelly, and L. J. Abu-Raddad, "Characterizing hepatitis C virus epidemiology in Egypt: Systematic reviews, meta-analyses, and meta-regressions," Scientific Reports, vol. 8, no. 1, p. 1661, 2018.

[3] S. Dasarathy and M. Merli, "Sarcopenia from mechanism to diagnosis and treatment in liver disease," Journal of Hepatology, vol. 65 , no. 6, pp. 1232-1244, 2016.

[4] M. A. Dunn, D. A. Josbeno, A. R. Schmotzer et al., "The gap between clinically assessed physical performance and objective physical activity in liver transplant candidates," Liver Transplantation, vol. 22, no. 10, pp. 1324-1332, 2016.

[5] E. J. Carey, D. E. Steidley, B. A. Aqel et al., "Six-minute walk distance predicts mortality in liver transplant candidates," Liver Transplantation, vol. 16, no. 12, pp. 1373-1378, 2010.

[6] P. Tandon, N. Tangri, L. Thomas et al., "A rapid bedside screen to predict unplanned hospitalization and death in outpatients with cirrhosis: a prospective evaluation of the clinical frailty scale," American Journal of Gastroenterology, vol. 111, no. 12, pp. 17591767, 2016.

[7] L. Zenith, N. Meena, A. Ramadi et al., "Eight weeks of exercise training increases aerobic capacity and muscle mass and reduces fatigue in patients with cirrhosis," Clinical Gastroenterology and Hepatology, vol. 12, no. 11, pp. 1920-1926, 2014.

[8] E. Román, M. T. Torrades, M. J. Nadal et al., "Randomized pilot study: Effects of an exercise programme and leucine supplementation in patients with cirrhosis," Digestive Diseases and Sciences, vol. 59, no. 8, pp. 1966-1975, 2014.

[9] M. Sinclair, M. Grossmann, R. Hoermann, P. W. Angus, and P. J. Gow, "Testosterone therapy increases muscle mass in men with cirrhosis and low testosterone: A randomised controlled trial," Journal of Hepatology, vol. 65, no. 5, pp. 906-913, 2016.

[10] H.-W. Chen and M. A. Dunn, "Arresting frailty and sarcopenia in cirrhosis: Future prospects," Clinical Liver Disease, vol. 11, no. 2, pp. 52-57, 2018. 
[11] K. Rockwood, X. Song, C. MacKnight et al., "A global clinical measure of fitness and frailty in elderly people," Canadian Medical Association Journal, vol. 173, no. 5, pp. 489-495, 2005.

[12] J. C. Lai, K. E. Covinsky, J. L. Dodge et al., "Development of a novel frailty index to predict mortality in patients with endstage liver disease," Hepatology, vol. 66, no. 2, pp. 564-574, 2017.

[13] E. B. Tapper, D. Finkelstein, M. A. Mittleman, G. Piatkowski, and M. Lai, "Standard assessments of frailty are validated predictors of mortality in hospitalized patients with cirrhosis," Hepatology, vol. 62, no. 2, pp. 584-590, 2015.

[14] J. C. Lai, M. L. Volk, D. Strasburg, and N. Alexander, "Performance-based measures associate with frailty in patients with endstage liver disease," Transplantation, vol. 100, no. 12, pp. 26562660, 2016.

[15] S. M. A. da Câmara, B. E. Alvarado, J. M. Guralnik, R. O. Guerra, and Á. C. C. Maciel, "Using the Short Physical Performance Battery to screen for frailty in young-old adults with distinct socioeconomic conditions," Geriatrics \& Gerontology International, vol. 13, no. 2, pp. 421-428, 2013.

[16] P. S. Kamath, R. H. Wiesner, M. Malinchoc et al., "A model to predict survival in patients with end-stage liver disease," Hepatology, vol. 33, no. 2, pp. 464-470, 2001.

[17] C. G. Child and J. G. Turcotte, "Surgery and portal hypertension," in The Liver and Portal Hypertension, C. G. Child, Ed., pp. 50-72, WB: Saunders, Philadelphia, PA, USA, 1st edition, 1964.

[18] J. M. Guralnik, E. M. Simonsick, L. Ferrucci et al., "A short physical performance battery assessing lower extremity function: Association with self-reported disability and prediction of mortality and nursing home admission," The Journals of Gerontology. Series A, Biological Sciences and Medical Sciences, vol. 49, no. 2, pp. M85-M94, 1994.

[19] H. C. Roberts, H. J. Denison, H. J. Martin et al., "A review of the measurement of grip strength in clinical and epidemiological studies: towards a standardised approach," Age and Ageing, vol. 40, no. 4, pp. 423-429, 2011.

[20] Y. Peng, X. Qi, and X. Guo, "Child-pugh versus meld score for the assessment of prognosis in liver cirrhosis," Medicine, vol. 95, no. 8, Article ID e2877, 2016.

[21] Y. Peng, X. Qi, S. Tang et al., "Child-Pugh, MELD, and ALBI scores for predicting the in-hospital mortality in cirrhotic patients with acute-on-chronic liver failure," Expert Review of Gastroenterology \& Hepatology, vol. 4124, pp. 971-980, 2016.

[22] N. Golse, P. O. Bucur, O. Ciacio et al., "A new definition of sarcopenia in patients with cirrhosis undergoing liver transplantation," Liver Transplantation, vol. 23, no. 2, pp. 143-154, 2017.

[23] L. Carvalho and E. R. Parise, "Evaluation of nutritional status of nonhospitalized patients with liver cirrhosis," Arquivos de Gastroenterologia, vol. 43, no. 4, pp. 269-274, 2006.

[24] M. Merli, M. Giusto, F. Gentili et al., "Nutritional status: its influence on the outcome of patients undergoing liver transplantation," Liver International, vol. 30, no. 2, pp. 208-214, 2010.

[25] P. Rita, J. Guralnik, J. C. Brown et al., "Short Physical Performance Battery and all-cause mortality: systematic review and meta-analysis," BMC Medicine, vol. 14, no. 1, pp. 1-9, 2016.

[26] M. A. Dunn, D. A. Josbeno, A. D. Tevar et al., "Frailty as tested by gait speed is an independent risk factor for cirrhosis complications that require hospitalization," American Journal of Gastroenterology, vol. 111, no. 12, pp. 1768-1775, 2016.

[27] E. S. Orman, M. Ghabril, T. W. Emmett, and N. Chalasani, "Hospital readmissions in patients with cirrhosis: a systematic review," Journal of Hospital Medicine, vol. 13, no. 7, pp. 490-495, 2018.

[28] I. Graupera, E. Solà, N. Fabrellas et al., "Urine monocyte chemoattractant protein-1 is an independent predictive factor of hospital readmission and survival in cirrhosis," PLOS ONE, vol. 11, no. 6, p. e0157371, 2016.

[29] S. Rassameehiran, C. Mankongpaisarnrung, G. Sutamtewagul, S. Klomjit, and A. Rakvit, "Predictor of 90-day readmission rate for hepatic encephalopathy," Southern Medical Journal, vol. 109, no. 6, pp. 365-369, 2016.

[30] B. P. Morales, R. Planas, R. Bartoli et al., "Early hospital readmission in decompensated cirrhosis: Incidence, impact on mortality, and predictive factors," Digestive and Liver Disease, vol. 49, no. 8, pp. 903-909, 2017.

[31] K. Agrawal, P. Kumar, R. Markert, and S. Agrawal, "Risk factors for 30-day readmissions of individuals with decompensated cirrhosis," Southern Medical Journal, vol. 108, no. 11, pp. 682687, 2015.

[32] A. G. Singal, R. S. Rahimi, C. Clark et al., "An automated model using electronic medical record data identifies patients with cirrhosis at high risk for readmission," Clinical Gastroenterology and Hepatology, vol. 11, no. 10, pp. 1335-1341, 2013.

[33] S. Le, T. Spelman, C.-P. Chong et al., "Could adherence to quality of care indicators for hospitalized patients with cirrhosisrelated ascites improve clinical outcomes?" American Journal of Gastroenterology, vol. 111, no. 1, pp. 87-92, 2016.

[34] K. Berman, S. Tandra, K. Forssell et al., "Incidence and predictors of 30-day readmission among patients hospitalized for advanced liver disease," Clinical Gastroenterology and Hepatology, vol. 9, no. 3, pp. 254-259, 2011.

[35] J. S. Bajaj, K. R. Reddy, P. Tandon et al., “The 3-month readmission rate remains unacceptably high in a large North American cohort of patients with cirrhosis," Hepatology, vol. 64, no. 1, pp. 200-208, 2016.

[36] L. Ferrucci, S. Bandinelli, E. Benvenuti et al., "Subsystems contributing to the decline in ability to walk: bridging the gap between epidemiology and geriatric practice in the InCHIANTI study," Journal of the American Geriatrics Society, vol. 48, no. 12, pp. 1618-1625, 2000.

[37] S. Studenski, S. Perera, K. Patel et al., "Gait speed and survival in older adults," JAMA, vol. 305, no. 1, pp. 50-58, 2011.

[38] N. M. Peel, S. S. Kuys, and K. Klein, "Gait speed as a measure in geriatric assessment in clinical settings: a systematic review," The Journals of Gerontology. Series A, Biological Sciences and Medical Sciences, vol. 68, no. 1, pp. 39-46, 2013. 


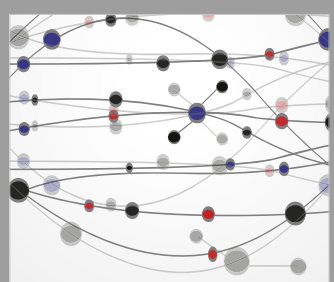

The Scientific World Journal
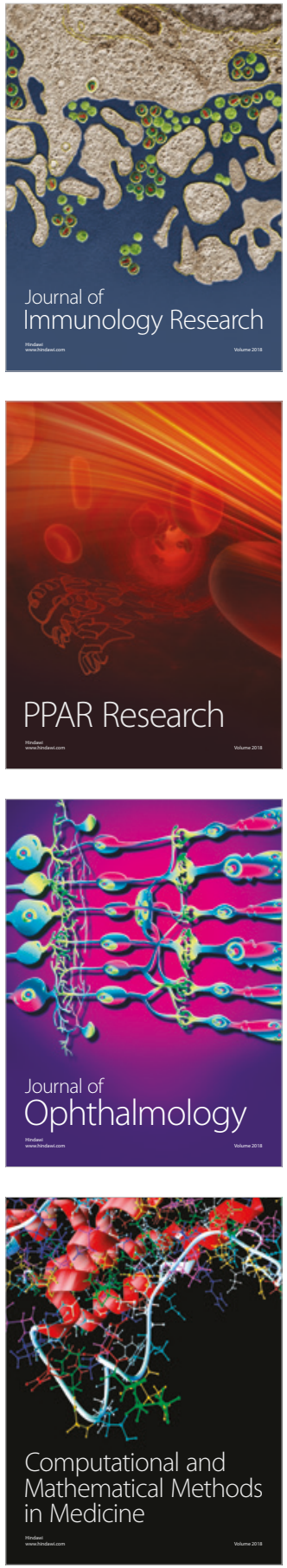

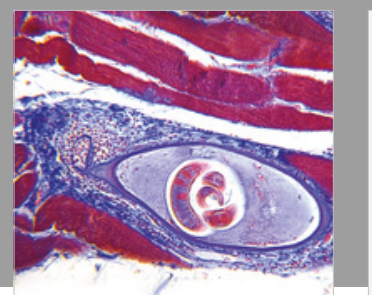

Gastroenterology Research and Practice

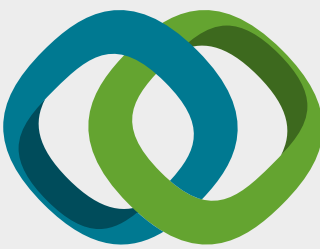

\section{Hindawi}

Submit your manuscripts at

www.hindawi.com
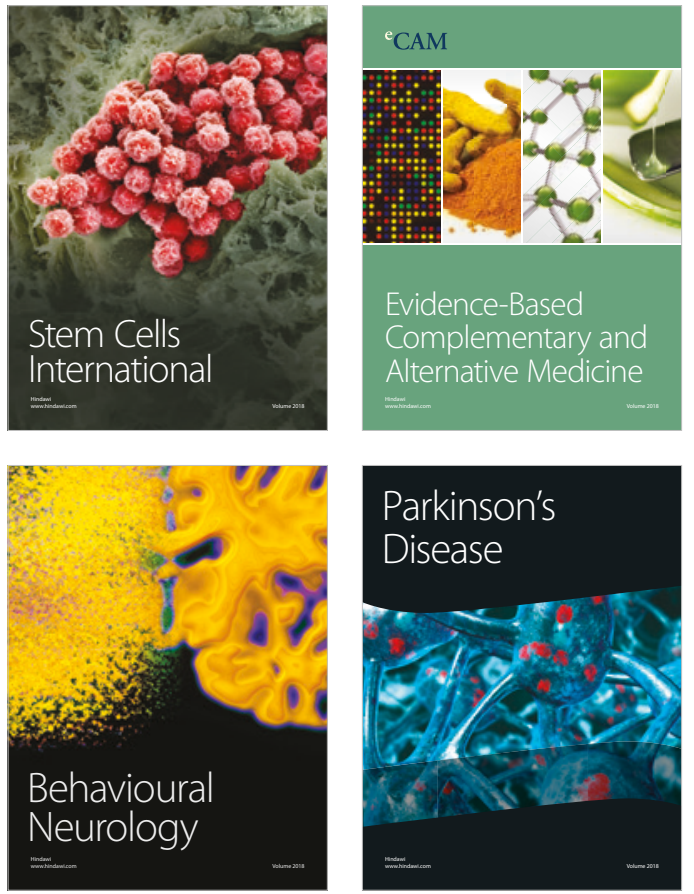

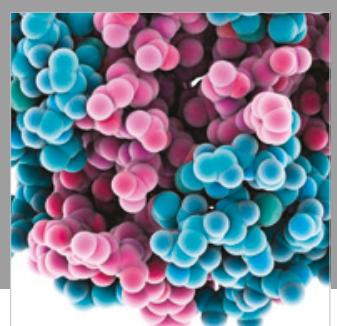

ournal of

Diabetes Research

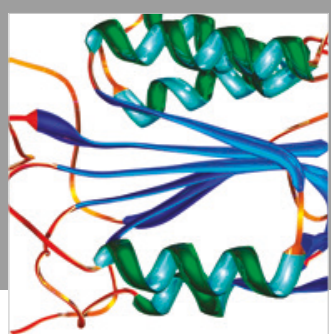

Disease Markers
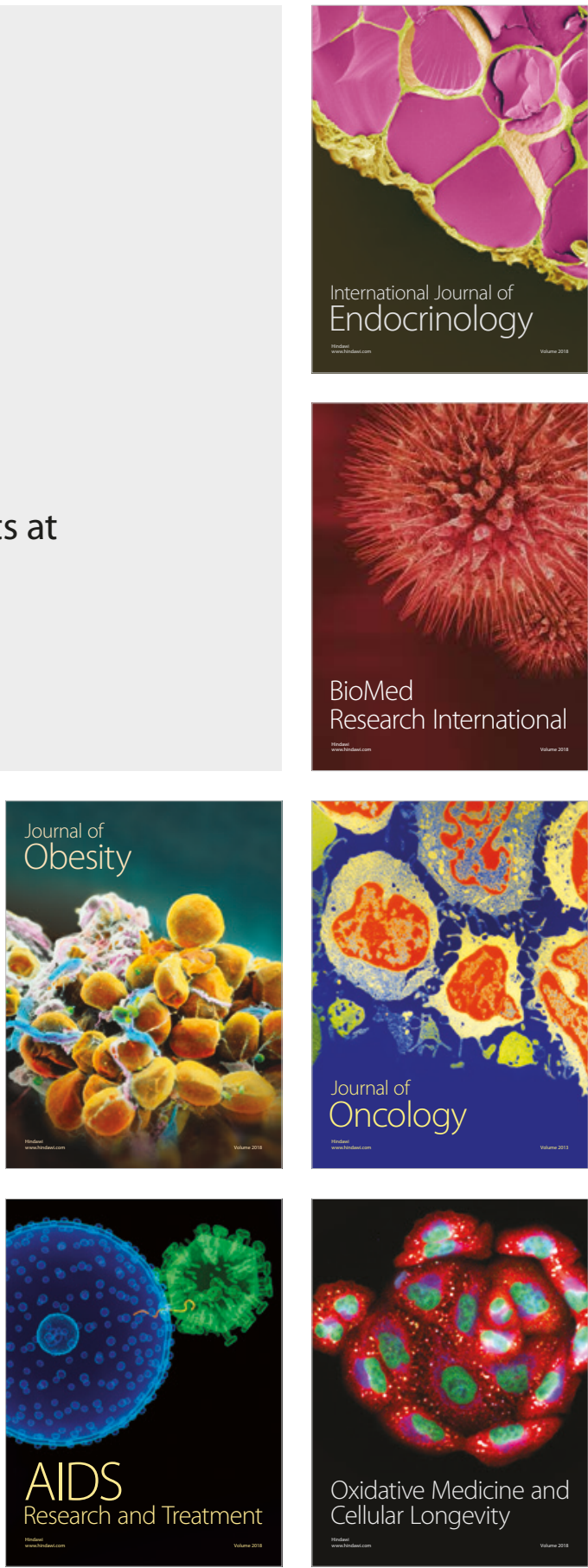